\title{
El Prácticum, un Espacio para la Investigación Transformadora en los Contextos Educativos Infantiles
}

\author{
The Practicum as a Context for Transformative Research in \\ Early Childhood Education
}

\author{
Rosario Mérida-Serrano * \\ María Elena González-Alfaya \\ María de los Ángeles Olivares-García \\ Julia Rodríguez-Carrillo \\ Miguel Muñoz-Moya \\ Universidad de Córdoba, España
}

\begin{abstract}
Esta investigación educativa se enmarca en un paradigma crítico. Con el objetivo de favorecer la puesta en marcha de procesos educativos transformadores, en la Red de Infantil Escuela-Centro de Profesorado-Universidad se configuran equipos híbridos de investigación en los que participan maestras, asesoras, profesorado y estudiantes universitarios. Se utilizan tres instrumentos de recogida y producción de información: dos grupos focales, 25 memorias reflexivas y 25 procesos de documentación pedagógica. Se utilizan técnicas de análisis del discurso (datos textuales) y análisis de contenido (datos visuales). Los resultados muestran los beneficios de la formación de equipos híbridos para evitar la rutinización en el aula y potenciar una teoría que emerge de la práctica. Como conclusiones más novedosas destacan: (1) la documentación pedagógica, al captar situaciones educativas, facilita la reflexión en y sobre la acción, haciendo posible repensar la práctica a la luz de la teoría; (2) la pareja educativa, al plantear una relación pedagógica simétrica, propicia un diálogo profesional en la que se recrea y confronta la teoría con las situaciones prácticas vividas.
\end{abstract}

Descriptores: Investigación social; Formación de docentes de preescolar; Práctica pedagógica; Educación de la primera infancia.

This educational research is framed within a critical paradigm. Pursuing the aim of developing transformative educational processes, and within the RIECU network (Early Childhood Education Schools-Center for Teacher Training-University), hybrid teams have been constituted, where early childhood teachers, advisors, university professors, and students participate. The study implements three different techniques for data collection and production: two focus groups, 25 reflective diaries and 25 processes of pedagogical documentation. An analysis of discourse (textual data) and content (visual data) has been used. The results show the benefits of hybrid teams for avoiding routinization in the classroom and for contributing to the development of an educational theory that emerges from practice. The most outstanding conclusions are: (1) pedagogical documentation that captures educational moments promotes reflection in-and-about the action and help teachers in rethinking their role by acknowledging what theory says; (2) the educational pair which entails a horizontal pedagogical relationship, fosters a professional dialogue where it is possible to re-create and confront the theory by means of the practical situations that have been experienced.

Keywords: Social research; Preschool teacher education; Teaching practice; Early childhood education.

*Contacto: ed1meser@uco.es

Recibido: $\quad 19$ de julio 2019

$1^{\text {a }}$ Evaluación: 2 de octubre 2019

ISSN: $1696-4713$

$2^{\text {a }}$ Evaluación: 3 de enero 2020

www.rinace.net/reice/

Aceptado: 11 de febrero 2020

revistas.uam.es/reice 


\section{Revisión de la literatura}

La comunidad científica no posee una percepción unívoca de las relaciones que se establecen en el triángulo formado por la teoría de la educación, la práctica y la investigación educativa (Álvarez, 2015; Denzin, 2017; Korthagen, 2010; Pérez, 2012).

En esta investigación se parte de un paradigma crítico de investigación educativa donde la teoría y la práctica se consideran dimensiones mutuamente constituyentes, que se hallan dialécticamente relacionadas (Zeichner y Liston, 2013). Como indican Colmenares y Piñero (2008) "se plantea la necesidad de asumir una concepción ontoepistémica, sociocrítica o socioconstructivista de la realidad social, en la cual se generan espacios por y entre los actores sociales para el diálogo, la reflexión y la co-construcción del conocimiento" (p. 96). Asumimos los principios que sugieren autores como Adorno (1991), Habermas (1999) y Horkheimer (2000) y que se resumen en: (1) Comprender la realidad como praxis; (2) Unir práctica y teoría, integrando conocimiento, acción y valores; (3) Orientar el conocimiento hacia una visión dialéctica y global de la realidad educativa; (4) Integrar a todos los participantes, incluyendo a la persona que investiga en procesos autorreflexivos para tomar decisiones consensuadas; y (5) Aceptar una visión democrática del conocimiento.

Dentro del paradigma crítico se opta por una metodología de investigación-acción colaborativa, asumiendo las aportaciones de Kemmis (2002) cuando indica:

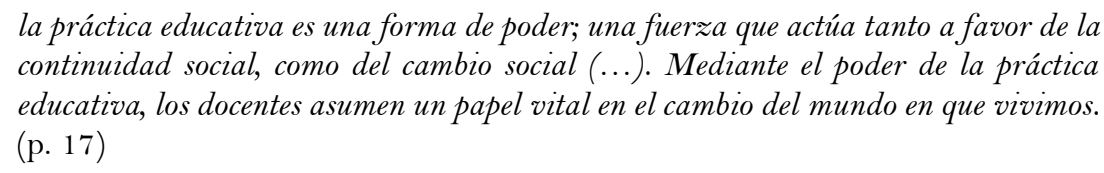

En este estudio se aboga por esta metodología porque, siguiendo las aportaciones de Cruger (2018): (1) El desafío de diseñar un Prácticum innovador, en el que participan de forma conjunta equipos híbridos de profesorado universitario, asesoras de Infantil y estudiantes de Ciencias de la Educación, surge de la comunidad en estudio; (2) Se trata de integrar y contextualizar los saberes de todas las personas que participan en la Red de Infantil Escuela-Centro de Formación del Profesorado-Universidad (en adelante, RIECU); (3) La investigación se desarrolla íntegramente en los centros educativos, en el contexto natural del aula; y (4) La construcción del saber pedagógico se efectúa a través de aprendizajes dialógicos adquiridos mediante relaciones horizontales entre los participantes.

En este trabajo se presenta y evalúa la experiencia del Prácticum III del Grado de Infantil de la Red de Infantil Escuela-Centro de Formación del Profesorado-Universidad (en adelante, RIECU) que incorpora, a través de una metodología de investigación-acción colaborativa, la documentación pedagógica y la pareja educativa como estrategias para potenciar el diálogo profesional y la reflexión en y sobre la acción.

\subsection{Relación entre investigación educativa transformadora e investigación-acción colaborativa}

El actual contexto social, neoliberal y globalizado, se refuerza y perpetúa con medidas tecnocráticas en las políticas educativas (Calvo y Susinos, 2010). De este modo, en más ocasiones de las deseables, se plantea en la formación inicial del profesorado, un enfoque orientado, prioritariamente, hacia la economía y el mercado profesional. Se omite de esta 
manera el debate sobre el sentido educativo, los fundamentos epistemológicos, axiológicos y metodológicos que ha de sustentar la investigación educativa (Rivas et al., 2012).

Nuestras convicciones, y compromiso social como investigadoras, nos acercan a una concepción epistemológica de la ciencia, entendida como una herramienta de transformación social que ha de saltar los muros de la universidad (Ruiz et al., 2018). No se puede seguir asumiendo un concepto de ciencia supuestamente neutral, alejada de los sujetos de investigación, que se diseña, piensa y 'empaqueta' en las instituciones de Educación Superior y se aplica en las escuelas y entidades sociales. Este modelo genera unos roles jerarquizados y crea una brecha de desigualdad que poco favorece la equidad, la justicia social y la corresponsabilidad (Denzin, 2017).

Optamos por una investigación educativa que, para ser transformadora, necesariamente debe asumir un enfoque inclusivo respecto a los saberes y la capacidad de producir conocimiento pedagógico de sus protagonistas, es decir los niños y niñas y sus docentes. Deseamos situarnos en una corriente contrahegemónica que permita repensar el 'pensamiento uniforme' y las prácticas rutinarias asentadas en una escuela transmisiva que perpetúa y reproduce las desigualdades. Hemos de tener en cuenta que la investigación educativa transformadora, como práctica social que es, puede contribuir a reproducir un sistema de organización, conocimientos, valores y actitudes que refuerce un sistema injusto o, por el contrario, convertirse en un instrumento para potenciar la formación de personas críticas y comprometidas con la transformación de una sociedad más justa, más igualitaria y más sostenible (Oliveros, 2018).

La investigación transformadora que se expone y analiza en este trabajo se apoya en procesos innovadores que generan un cambio en el modo de representar, interpretar y repensar el hecho educativo, considerándolo como un acto social, cultural, epistemológico y político (Arnaus y Piussi, 2010; Barnett, 2002, 2008; Carr, 2002; Colmenares, 2012).

Como se indicó previamente, el paradigma crítico y socioconstructivista en el que se enmarca este estudio se concreta en una metodología de investigación-acción colaborativa (Inayatullah y Gidley, 2003; Zamboni, 2009; Zeichner, 2010). Se asume el concepto de investigación-acción colaborativa que proponen Boggino y Rosekrans (2007):

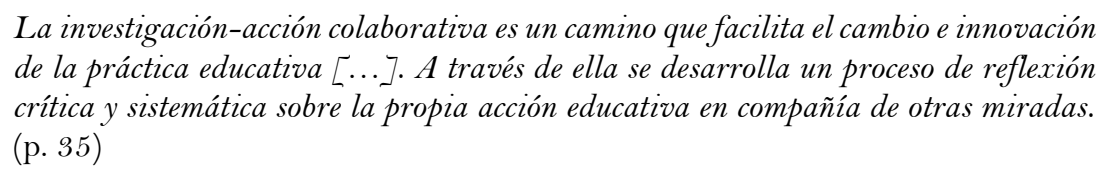

La investigación-acción colaborativa es un camino que facilita el cambio e innovación de la práctica educativa [...]. A través de ella se desarrolla un proceso de reflexión crítica y sistemática sobre la propia acción educativa en compañía de otras miradas. (p. 35)

\subsection{La investigación-acción colaborativa mediante equipos híbridos escuela- universidad}

Un proceso de investigación-acción colaborativa debe trascender la actual separación entre la teoría y la práctica, abogando por una epistemología de la práctica y una revalorización del saber experiencial de los y las docentes (Korthagen, 2010). Por ello, en esta investigación se suscribe una concepción de la investigación educativa como una palanca de transformación social que modifica las relaciones tradicionales entre la universidad y la escuela (Sancho-Gil, 2017).

Una forma de superar la brecha entre teoría y práctica, entre escuela y universidad, es crear equipos híbridos de investigación en los que se definan todas las fases del proceso investigador de forma consensuada. Con esta intención nace RIECU, que es una red compuesta por un grupo de profesionales vinculadas al Aprendizaje Basado en Proyectos 
(en adelante, $\mathrm{ABP}$ ) que pretenden investigar sobre el impacto que tiene el ABP en el desarrollo y aprendizaje de la infancia, así como en las competencias docentes que se desarrollan al participar en una red profesional de Educación Infantil (Krichesky y Murillo, 2011).

Como punto de partida, el equipo consensua una serie de principios sobre el tipo de investigación educativa que desea desarrollar. Dichos principios son:

- Selección de temas de investigación que emerjan de la escuela y respondan a una necesidad de la comunidad educativa. De esta forma se garantiza la utilidad social de la investigación educativa.

- Configuración de un equipo de investigación híbrido, en el que docentes de Educación Infantil y profesorado universitario asumen roles de investigación simétricos. Se siguen las propuestas de Zeichner (2010), al considerar necesario "desarrollar espacios híbridos, o un tercer espacio formativo, en el que se propicie un aprendizaje no jerárquico que ponga en relación el conocimiento teórico y práctico" (p. 135) y en el que participen las profesionales de las escuelas junto con el profesorado universitario.

- Elección consensuada del paradigma de investigación en el que nos situamos. La opción seleccionada es el paradigma crítico, por tener una incidencia directa en la transformación de la realidad investigada (Rivera-Vargas, Sánchez-Valero y Sancho-Gil, 2019).

- Negociación de cada uno de los elementos y fases de la investigación mediante un proceso dialógico de ajuste y complementación de miradas.

- Elección, a demanda de las docentes, de la investigación-acción como metodología para comprender, transformar y mejorar los contextos prácticos.

- Corresponsabilidad en la negociación del informe de investigación y la difusión de los resultados.

- Asunción de unos principios éticos sobre los que se sustenta la investigación con la infancia.

Estos principios de actuación son fruto de un proceso de interacción y acuerdos personales y profesionales que no han estado exentos de dificultades, de avances, de retrocesos y de cuestionamientos permanentes.

\subsection{El diálogo profesional y la reflexión sobre la acción a través de la documentación pedagógica y la pareja educativa}

La investigación-acción colaborativa, desarrollada en este trabajo, se apoya en el uso de la pareja educativa y la documentación pedagógica, como herramientas para favorecer un diálogo profesional y una reflexión sobre la práctica (Farrell, 2018; Schön, 1992).

Podríamos preguntarnos en qué medida la pareja educativa favorece un diálogo que haga posible la reflexión sobre la acción. En efecto, la pareja educativa se concibe como un encuentro de personas, vinculadas a la docencia que, a través del diálogo, recrean y reconstruyen la experiencia pedagógica vivida (Cotrina, García y Caparrós, 2017). Se entiende por diálogo profesional, coincidiendo con Urdaneta (2014), un intercambio comunicativo caracterizado por: (1) Horizontalidad entre los interlocutores para asegurar la expresión libre; (2) Argumentación basada en evidencias empíricas y fundamentos 
teóricos; y (3) Apertura y empatía para incorporar posiciones discrepantes. Este diálogo profesional, puesto en marcha por la pareja educativa, facilitará una reflexión en y sobre sobre la acción, a partir del análisis, cuestionamiento, recreación y confrontación con la teoría de las situaciones prácticas vividas. De acuerdo con Schön (1992) y Perrenoud (2004) la reflexión en y sobre la acción permite preguntarse sobre lo que está pasando, valorar la mejor estrategia, comparar la acción con modelos teóricos y reconducir los aspectos desajustados de la práctica. A través de la reflexión, en definitiva, se puede teorizar la práctica y practicar la teoría, apostando por una relación compleja y dialéctica entre ambas (Pérez, 2013).

En el Prácticum RIECU, se organizan parejas educativas -integradas por la maestra/tutora profesional y la estudiante en prácticas- concebidas como un par de personas que poseen conocimientos docentes, cuyo nivel de desarrollo profesional y experiencia es diferente, pero que comparten una concepción de infancia y una valoración positiva de la metodología de ABP, lo cual propicia un diálogo profesional y una reflexión en y sobre la acción.

Otra de las herramientas que se utiliza en esta investigación, con el objetivo de incrementar la reflexión sobre la acción, es la documentación pedagógica. La documentación pedagógica consiste, siguiendo las aportaciones de Hoyuelos (2006, 2007) en la recogida y exposición sistemática y estética (a través de escritos, imágenes, paneles, vídeos, palabras de los niños y niñas, productos gráficos...) de los procesos educativos. No se trata solo de plasmar lo acontecido, sino de construir -en diversos formatos- un producto público que dé cuenta narrada de lo vivido y que ayude a hacer visibles nuestros pensamientos, ideas e interpretaciones del aprendizaje. Responde a un plan detallado de observación y escucha de la infancia, cuya finalidad es entender la cultura infantil: su forma de pensar, hacer, preguntar, teorizar o desear. Escuchar significa estar atento, con todos los sentidos, a reconocer todos los lenguajes de la infancia en su relación con el mundo, es un proceso para desarrollar la sensibilidad educativa, que se aprende y se hace fundamentalmente en grupo. Escuchar es una actitud que nos debe remover desde dentro para vaciarnos de prejuicios. Es romper para construir.

\subsection{La Red de Infantil Escuela-Centro de Formación Permanente del Profesorado- Universidad (RIECU), un contexto para la investigación-acción colaborativa}

La Red RIECU empieza a gestarse en el curso 2007/08. Dos circunstancias favorecen el inicio de una colaboración más estable entre la escuela, el CEP y la Universidad: (1) la implantación del Espacio Europeo de Educación Superior, en el que se incluye la necesidad de trabajar por competencias en la universidad; (2) la oportunidad que se ofrece desde el CEP de Córdoba para abordar procesos colaborativos entre la formación docente inicial y continua, tal y como se recoge en el III Plan de Formación Permanente del Profesorado (2013), elaborado por la Consejería de Educación y Ciencia de la Junta de Andalucía. En este documento se indican las competencias docentes profesionales que debe poseer el profesorado y se señala que uno de los objetivos de su formación inicial docente ha de ser "reflexionar críticamente sobre su práctica docente en su ejercicio profesional, a través de procesos de autoevaluación, que le permitan contrastarla con otras experiencias y utilizar sus resultados para mejorar" (art. 4.b, p. 9).

En este contexto normativo, favorable para la investigación educativa, se consolida la Red RIECU, sustentada en el interés compartido que tienen las tres instituciones que la conforman (escuelas, CEP y universidad) para investigar sobre el ABP, el cual se concibe 
como una forma alternativa de entender la escuela infantil. El ABP es abordado desde la formación teórica que se imparte en las aulas universitarias y, paralelamente, es ofrecido como objeto de aprendizaje en la formación permanente. Este 'cruce de caminos' ofrece un espacio favorable para iniciar procesos de investigación-acción liderados por equipos de investigación híbridos integrados por maestras, asesoras y profesoras universitarias.

Es el Prácticum un contexto de intersección que actúa como bisagra entre la formación docente inicial y continua. Resulta un momento muy apropiado para introducir procesos de investigación-acción colaborativos liderados por equipos híbridos de maestras, asesoras, profesoras y estudiantes. En dichos equipos se repiensa el papel de la investigación educativa, concibiéndola como un espacio donde se contrasta la teoría y la práctica, donde emerge y se genera conocimiento que surge de la propia acción. El prácticum RIECU incluye tres elementos transformadores: (1) los procesos de investigación-acción colaborativa, (2) la documentación pedagógica y (3) la pareja educativa.

\section{Método}

El problema que guía este trabajo es analizar si la investigación-acción colaborativa que se desarrolla en el prácticum RIECU, a través de la pareja educativa y la documentación pedagógica, se puede considerar una investigación educativa transformadora. Para ello se parte de tres interrogantes de investigación:

- ¿Contribuyen los procesos de investigación-acción colaborativos desarrollados en el Prácticum RIECU a transformar la práctica educativa?

- ¿Facilita la documentación pedagógica realizada en el Prácticum RIECU la reflexión en y sobre la práctica?

- ¿Propicia la pareja educativa un diálogo profesional para mejorar y transformar la práctica educativa?

\section{Participantes}

Durante el curso 2018/19 han participado, de forma voluntaria, en el Prácticum RIECU 24 maestras y 1 maestro del segundo ciclo de Educación Infantil que imparten docencia en centros públicos de Córdoba capital y provincia, 25 estudiantes de $4^{\circ}$ curso del Grado de Infantil, 5 investigadores del Departamento de Educación de la Universidad de Córdoba, 2 asesoras del CEP de Córdoba, un total de 57 participantes.

\section{Instrumentos de recogida y producción de información}

Se utilizan tres instrumentos para obtener una triangulación metodológica que apoye la credibilidad y veracidad de las conclusiones: (1) Grupo focal (Krueger, 1994) (uno con 4 maestras, 2 asesoras y 2 profesoras universitarias), y otro con 8 estudiantes universitarias. Se dividen ambos colectivos para facilitar la expresión libre de las estudiantes; (2) Documentación pedagógica (Hoyuelos, 2007), realizada por las estudiantes durante su estancia en los centros de prácticas; e (3) Informe reflexivo de prácticas (25), realizados por las estudiantes. Se dispone, por tanto, de datos textuales y visuales. 


\section{Técnicas de análisis, categorización y codificación}

Se utilizan dos técnicas: (1) análisis del discurso (Wodak, 2000), para las opiniones expresadas en los grupos focales y en los informes reflexivos de prácticas y (2) análisis de contenido (Espín, 2002) para clasificar, por afinidad temática, las evidencias visuales recogidas en la documentación pedagógica.

Para el análisis del discurso los datos se transcriben, clasifican, codifican y sintetizan aplicando un proceso de categorización inductivo que emerge de la voz de los protagonistas. Se establecen dos tipos de unidades de análisis: (1) dimensiones, que son unidades de análisis macro vinculadas a los grandes ejes del Prácticum RIECU; y (2) categorías, que son unidades de análisis micro, que recogen la palabra de los participantes. Para el análisis de contenido de las imágenes recogidas en la documentación pedagógica se realiza una clasificación por temáticas afines según las dimensiones establecidas, se codifican y se utilizan para apoyar y complementar el discurso de los participantes.

Se ha realizado una codificación de los datos textuales y visuales, aplicando el siguiente procedimiento: en primer lugar, se incluyen las iniciales del instrumento utilizado: GF, grupo focal; MR, memoria reflexiva y DP, documentación pedagógica, seguido de la primera letra de cada colectivo, Maestras-Estudiantes-Asesoras-Profesoras universitarias y, al final, se asigna un número a cada participante de los diferentes colectivos. De este modo el código GFM14 hace referencia a la intervención en el grupo focal de la maestra a la que se ha asignado el número 14 .

\section{Triangulación}

Además de la triangulación metodológica, para el proceso de categorización, se aplica un acuerdo interjueces en el que interviene una representante de cada colectivo -maestra, asesora, estudiante y profesora- y, mediante un diálogo intersubjetivo, se aceptan las categorías coincidentes y se desechan las discrepantes.

\section{Resultados}

Los datos se ordenan en la siguiente matriz hermenéutica, diseñada con las aportaciones de todos los colectivos participantes en la investigación. Es una estructura analítica donde se clasifican las intervenciones y se ponen a dialogar las aportaciones de los diferentes participantes de una misma dimensión (cuadro 1).

Cuadro 1. Matriz hermenéutica

\begin{tabular}{ll}
\hline \multicolumn{1}{c}{ DIMENSIONES } & \multicolumn{1}{c}{ CATEGORÍAS } \\
\hline & 1.1. Tiempo para la reflexión \\
& 1.2. Analizar con una mirada externa \\
1. Investigación-acción colaborativa & 1.3. Evaluar para mejorar \\
& 1.4. Cambiar desajustes sobre la marcha \\
& 1.5. Aprendizaje continuo \\
\hline \multirow{2}{*}{ 2. Documentación pedagógica } & 2.1. Selección de situaciones relevantes \\
& 2.2. Escucha personalizada al alumnado infantil \\
& 2.3. Interacción entre iguales \\
\hline \multirow{3}{*}{ 3. Pareja educativa } & 3.1. Alianza profesional \\
& 3.2. Complementariedad de miradas \\
& 3.3. Discrepancias de estilos educativos \\
\hline
\end{tabular}

Fuente: Elaboración propia. 
En la categoría 1.1. de la primera dimensión, referida a la investigación-acción colaborativa, existe coincidencia entre los diferentes colectivos implicados en que propicia un tiempo para la reflexión. Reservar una hora y media semanal, sin atención a niños y niñas, permite tener un tiempo de reposo que hace posible la reflexión en y sobre la práctica del equipo híbrido formado por maestras, asesoras, profesorado y estudiantes. No significa que la reflexión solo se lleve a cabo en este tiempo reservado. En el aula existen múltiples momentos donde se comenta, valora y realizan análisis entre la maestra y la estudiante de prácticas, pero es una conversación poco sistemática, que está mediatizada por las múltiples demandas simultáneas que se producen en los contextos prácticos infantiles. Es un momento idóneo para aproximar teoría y práctica a través de la reflexión. Las participantes en esta investigación lo expresan del siguiente modo:

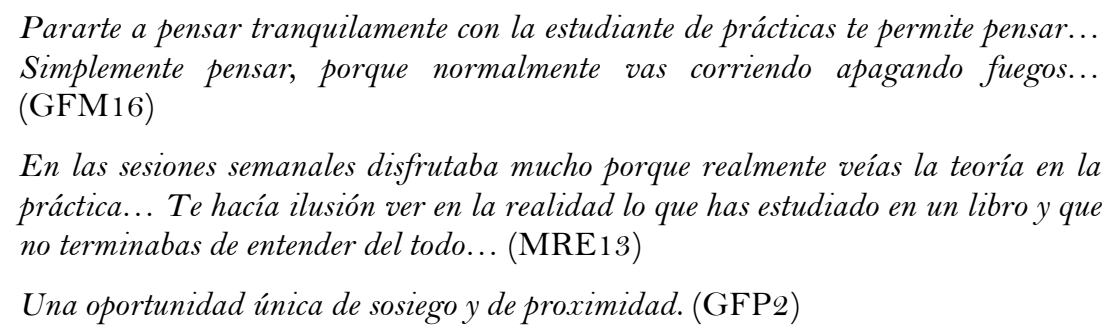

Nos viene bien a todas hablar tranquilamente... (GFA1)

Las estudiantes afirman que es un procedimiento adecuado para entender en la acción algunos conceptos que resultan incomprensibles en el plano teórico abstracto y que se pueden palpar cuando se concretan en el aula, con el asesoramiento de las maestras, que les ayudan a establecer relaciones entre la práctica y la teoría. Estos resultados refuerzan los hallazgos mostrados en investigaciones como las de Brydon-Miller, Greenwood y Maguire (2003) y Mérida, González y Olivares (2012). También resultan congruentes con la idea de reconstruir una 'teoría de la práctica', como sugieren Woolf y Wamba (2019), y expresa Whitehead (2008): "A living theory is an explanation produced by an individual for their educational influence in their own learning, in the learning of others, and in the learning of the social formation in which they live and work" (p. 104).

La segunda categoría de la primera dimensión se centra en analizar con una mirada externa la realidad educativa (figura 1). Tres de los colectivos consultados -profesoras, asesoras y maestras- expresan que los procesos de investigación-acción colaborativos permiten realizar un análisis desde fuera, aportando puntos de vista diferentes sobre una misma realidad. Se perciben matices que pasan desapercibidos para quien está cotidianamente en las aulas y rutiniza muchas dimensiones, asumiéndolas como algo normal. Siguiendo sus palabras:

Claro, mi punto de vista es diferente al de las maestras... cuando estás en el 'tajo' el árbol, a veces, te impide ver el bosque... A mí también me pasa en mis clases de la facultad. (GFP3)

Está claro que cuando estoy en la vorágine es difícil ser juez y parte... Me meto tanto... que pierdo la objetividad y la distancia... (GFM3) 


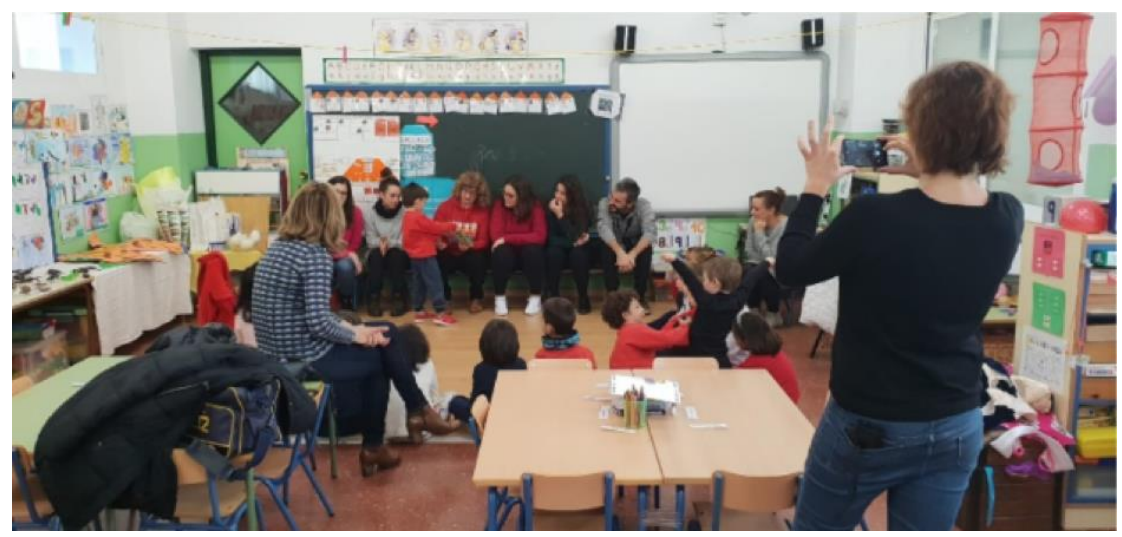

Figura 1. Maestra, profesorado, familias y niños y niñas valoran el proyecto mientras la estudiante documenta (DPE 18)

Fuente: Elaboración propia.

El trabajo de Suárez (2002) y de Colmenares y Piñero (2008, p. 96) también inciden en la aportación que hacen los agentes externos "para desarrollar el pensamiento práctico de los docentes, hacer uso de la reflexión y el diálogo, transformar ideas y ampliar la comprensión". En definitiva, como indican Miño Puigcercós, Domingo-Coscollola y Sancho-Gil (2019, p. 152), "Participatory action research (...) has proven to be appropriate way to promote and document the transformation of a teaching and learning culture".

La tercera categoría de la primera dimensión versa sobre la posibilidad que ofrecen los procesos de investigación-acción colaborativos de evaluar para mejorar. Es una opinión compartida por las profesoras y las estudiantes de universidad. Las cuales expresan una concepción formativa y global de la evaluación. Una evaluación que lleva implícita la intención de mejorar. Su opinión la expresan de la siguiente manera:

\footnotetext{
En los procesos de investigación-acción evaluamos colectivamente cómo va el proyecto. Pero una evaluación para que mejoremos todos... allí no vamos en plan inspectoras... (GFP3)
}

La cuarta categoría de la primera dimensión alude a cambiar desajustes sobre la marcha. Esta categoría incluye la opinión unánime de todos los colectivos implicados. Tanto las estudiantes y profesoras universitarias, como las maestras y asesoras, manifiestan que participar en los procesos de investigación-acción colaborativos les ofrece la oportunidad de detectar desajustes en el desarrollo de la práctica, e intervenir inmediatamente para reconducirlos. Esta retroalimentación constante permite atajar disfunciones cuando ocurren, evitando su continuidad y permanencia. Sus voces, en este sentido, dicen lo siguiente:

Lo mejor es que tienes la oportunidad de revertir rápido tus errores... que ahora al principio son muchos... (MRE6)

La investigación-acción te permite no solo ver los fallos, sino ponerle remedio de inmediato... (GFM7)

Reconducir los errores rápidamente te permite, además de no equivocarte más, atreverte a meterte en nuevos retos con la tranquilidad de que puedes rectificar en cualquier momento... (GFP3)

Estos hallazgos son congruentes con la tradición de las escuelas infantiles de Reggio Emilia y Losky (Altimir, 2010; Hoyuelos, 2004), y con la tradición investigadora que 
defiende la pareja educativa por su potencial como estrategia de apoyo en el aula (Cotrina, García y Caparrós, 2017; Friend et al., 2010), orientada a ofrecer una respuesta educativa más ajustada al alumnado que precisa una atención particular, ya sea puntual o sostenida en el tiempo (Chanmugam y Gerlach, 2013; Rodríguez y Grilli, 2013; Salamanca, 2011).

La quinta y última categoría de la primera dimensión hace alusión al aprendizaje continuo que se produce al participar en procesos de investigación-acción colaborativa. Es una categoría que la suscriben las maestras y las profesoras universitarias. Indican que la revisión continua de la práctica hace posible un aprendizaje permanente, analizando detalles que habitualmente se normalizan, y sintiendo cómo estos procesos le ayudan a su desarrollo profesional. Lo expresan del siguiente modo:

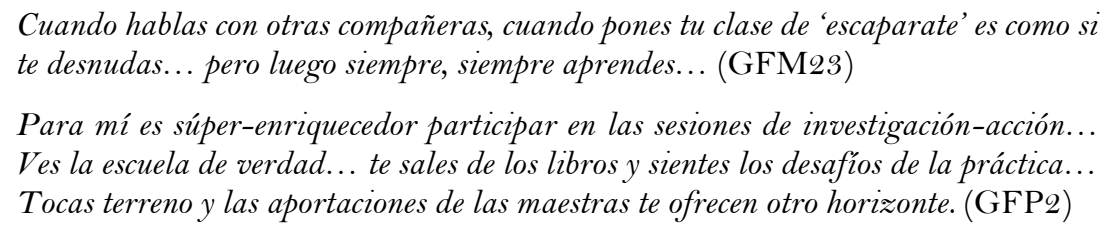

La segunda dimensión se refiere a la documentación pedagógica (figura 2). La primera categoría alude a la selección de situaciones relevantes que exige la aplicación de esta herramienta de investigación en las aulas infantiles. Expresan la dificultad para analizar la práctica y discernir lo esencial de lo accesorio, lo fundamental de lo superfluo. Supone un aprendizaje y un reto para las estudiantes adquirir la competencia para analizar la práctica e identificar los elementos fundamentales que la definen. Tienen que vivir un proceso de entrenamiento, apoyado por la maestra, para ir mejorando su capacidad de observación y de captación de las situaciones más relevantes. Convergen en una apreciación similar de esta categoría todos los colectivos implicados en la investigación:

A mí, la verdad, me costó trabajo eso de documentar. Primero no sabía qué tenía que fotografiar y qué no. Estaba todo el día con la cámara obsesionada... La segunda semana me di cuenta de que tenía que resumir y seleccionar lo más importante. (MRE15)

Lo de la documentación pedagógica es importante porque las obliga (a las estudiantes) a estar atentas a todo lo que ocurre en clase... Y también a diferenciar lo importante de lo que no lo es tanto... Al principio les cuesta, pero van cogiendo la dinámica poquito a poco. (GFM21)

La documentación pedagógica es una herramienta difícil de manejar... Es muy fácil echar fotos con el móvil... pero de ahí a documentar hay una gran distancia... Lo de menos es la parte técnica, lo difícil es seleccionar pedagógicamente lo fundamental y elaborar un discurso gráfico para reconstruir la práctica. (GFP2)

Documentar en Infantil es muy importante... Te permite centrarte en lo más relevante y a las estudiantes las orienta mucho para extraer lo importante de sus experiencias en las clases. (GFA 1 )

En este mismo sentido se pronuncia el Equipo Directivo y Educativo de la EEI La Alegría de la Huerta (2018) cuando expresan:

La documentación pedagógica nos ha permitido observar con más entusiasmo a las niñas y los niños, descubrir junto con ellos nuevas formas de interaccionar con las personas y con los objetos; nos ha permitido compartir cada experiencia con la pareja educativa y comparar nuestras miradas sobre los procesos de aprendizaje. (p. 3)

Nuestros hallazgos también son congruentes con las aportaciones de Rinaldi (2011, p. 13), cuando subraya la importancia de la documentación para "hacer visible los procesos de 
aprendizaje y las estrategias utilizadas por cada niño, sin embargo, siempre en un modo parcial y subjetivo".

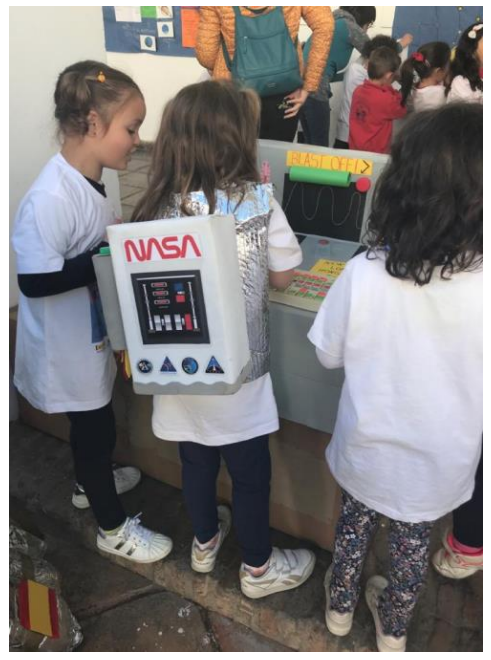

Figura 2. Documentación pedagógica del proyecto el Universo (DPE15)

Fuente: Elaboración propia.

La segunda categoría de la segunda dimensión se refiere a la escucha personalizada al alumnado de Educación Infantil. Las maestras indican que al documentar hay que focalizar mucho sobre las necesidades y evolución personal de cada discente. Que se deben captar momentos en los que cada niño o niña exprese, a través de su imagen y lenguaje corporal, las emociones y las interacciones con los objetos y con sus iguales (ver figura 3). Por su parte las estudiantes consideran muy complejo delimitar las individualidades de 25 pequeños y pequeñas interactuando simultáneamente. Ellas manifiestan que:

\begin{abstract}
Lo mejor de la documentación es saber captar esos momentos mágicos que se producen en el aula... Es tan difícil... $\Upsilon$ muchas veces tienen que ver con la cara de un niño, con sus ojos, su mirada... son expresiones de la cara y posturas espontáneas que reflejan nítidamente la sorpresa, la tristeza, el asombro... y tantas otras emociones. (GFM19)

A mí me cuesta trabajo...porque estoy más pendiente de hacer cosas con los niños y de apagar mil fuegos que hay en la clase, que de sacar fotos o vídeos de un niño... Cuando veo algo genial y voy a fotografiarlo ya ha pasado. (MRE7)
\end{abstract}

La tercera categoría de la segunda dimensión alude a la interacción entre iguales. Las estudiantes se autoperciben con más competencia para reflejar en sus documentaciones las situaciones grupales que la evolución personal de los discentes. También las maestras señalan que recoger los momentos clave de aprendizaje personal de cada alumno o alumna requiere un mayor entrenamiento para aprender a identificar las conductas y comportamientos donde se producen dichos momentos críticos. Por esta razón, y porque en la metodología de ABP la mayoría de las propuestas de aprendizaje tienen un marcado carácter colectivo, es por lo que prevalecen las imágenes que captan situaciones de aprendizaje colaborativo. Las estudiantes señalan que les sorprende más las interacciones educativas que se producen entre iguales que el formato interactivo en el que interviene la docente y un niño o varios.

To cuando estaba en la sesión reflexiva de pronto me daba cuenta de que la mayoría de las imágenes que tenía eran de situaciones de grupo de los niños. (MRE9) 
Es que verlos hablar, ayudarse y cómo razonaban y resolvían problemas juntos siendo tan pequeños era una gozada. Me ponía a echar fotos y vídeos sin parar. (GFE3)

A nuestras estudiantes les gusta mucho documentar... van siempre con sus móviles $y$ sobre todo se fijan en momentos grupales. (GFM4)

A ellas les cuesta un poco todavía ver cuándo hay un niño que está atrancado o que demanda un apoyo... Están más preocupadas por el control del grupo, porque no se les vaya de las manos... Es normal por ahí hemos pasado todas. (GFM1)

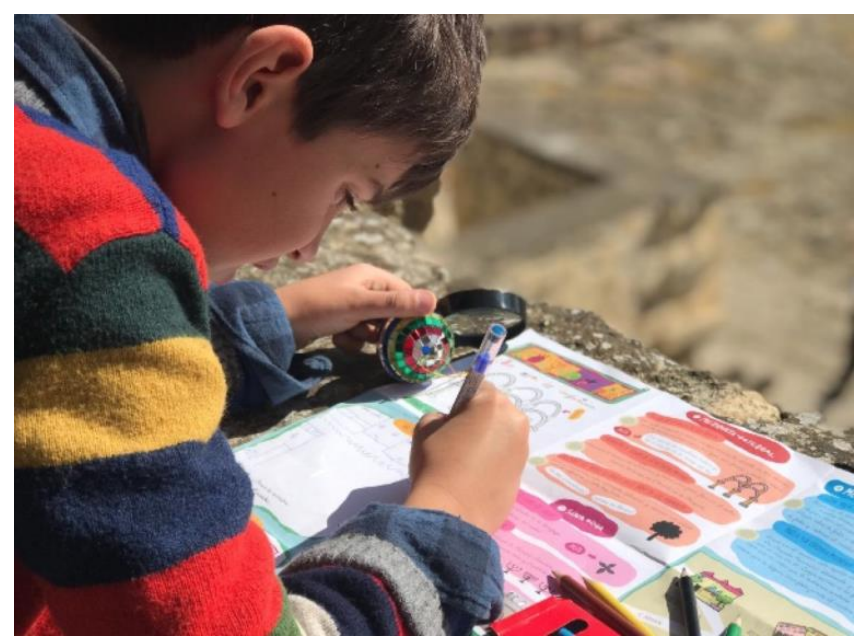

Figura 3. Documentación pedagógica. Trabajo personal (DPE11) Fuente: Elaboración propia.

La tercera y última dimensión incluye la pareja educativa. Se refiere a la relación que se establece entre la estudiante de prácticas y la docente. Es una relación que comienza a gestarse desde antes de la estancia de las alumnas y alumno en el centro. En las aulas universitarias se trabaja el concepto de pareja educativa y se indican los roles que ha de asumir el estudiantado. Para tener una primera aproximación entre ambas partes de la pareja educativa, se propicia al menos un encuentro antes de estar juntas en el aula de Educación Infantil. El tipo de interacción profesional que se aconseja es una modalidad simétrica, donde el aprendizaje dialógico es un eje de aprendizaje mutuo.

La primera categoría de la tercera dimensión hace referencia a la alianza profesional que se crea en la pareja educativa. Existe una amplia variabilidad de opiniones, dependiendo de la compatibilidad entre la identidad personal y profesional de quienes integran las parejas educativas, cuya configuración se realiza al azar. Veamos sus opiniones:

Lo mejor de la pareja es que te da mucho espacio la maestra... No sé no te sientes como estudiante, es como si tú también fueras ya maestra. (MRE17)

To he notado mucha diferencia con respecto al año pasado... He pasado de aprendiz a maestra novata... (risas). (GFE19)

Lo de la pareja está bien, las chicas vienen con una actitud más activa... $\Upsilon$ así se crean unos lazos profesionales y una complicidad fuerte... En la clase somos dos docentes... Y eso es muy bueno. (GFM4)

Hacemos piña... Este año ha sido fantástico... Íbamos las dos a una... Nos entendiamos sin hablarnos. (GFM20)

Cada año las maestras nos devuelven la idea de que las alumnas van mejor preparadas. En esto tiene mucho que ver la asignatura de Estrategias Educativas en Educación Infantil. (GFP1) 
Las maestras nos dicen que las parejas, en algunos casos, les sirven de estímulo. (GFA2)

Estos hallazgos son congruentes con la tradición de las escuelas infantiles de Reggio Emilia y Losky (Altimir, 2010; Hoyuelos, 2004), y con la tradición investigadora que defiende la pareja educativa por su potencial como estrategia de apoyo en el aula (Cotrina, García y Caparrós, 2017; Friend et al., 2010), orientada a ofrecer una respuesta educativa más ajustada al alumnado que precisa una atención particular, ya sea puntual o sostenida en el tiempo (Chanmugam y Gerlach, 2013; Rodríguez y Grilli, 2013; Salamanca, 2011).

La segunda categoría de la tercera dimensión alude a la complementariedad de miradas. Las maestras y las estudiantes resaltan la compañía y apoyo mutuo que experimentan como docentes al situarse en pareja en el aula. Tener la posibilidad de analizar la práctica confrontando dos puntos de vista, tener ayuda cuando se necesita, tener disponibilidad para ofrecer apoyos y ayudas educativas más personalizadas al alumnado, o atreverse a explorar nuevos territorios, son algunas de las 'ganancias' derivadas de la pareja educativa. Sus voces lo expresan con las siguientes palabras:

\begin{abstract}
Yo, la verdad, me he sentido muy acompañada y apoyada con mi maestra... Cuatro ojos siempre ven más que dos. (GFE13)

Cuando hacía el análisis que nos pedían en el prácticum la maestra me aportaba un montón de cosas que yo sola no veía. (GFE22)

Me encantaba cuando le proponía alguna actividad a mi maestra y me daba su opinión... Siempre mejoraba mi propuesta y me sentía más segura para hacerla. (MRE11)

Hemos hecho cosas muy chulas... Cosas que yo sola no me hubiera atrevido... Es como un ¡Venga... Nosotras podemos! (GFM18)

La chica de prácticas ha ayudado mucho a los niños que tenían algún problema. (GFM19)
\end{abstract}

La última dimensión de la tercera categoría incluye las discrepancias de estilos educativos que se han percibido en algunas parejas educativas. Estilos educativos que se definen básicamente por: (1) los rasgos de personalidad de la maestra y la estudiante de prácticas; (2) por su manera de concebir la Educación Infantil; y (3) por la concepción de infancia que poseen. La convivencia en el aula de dos identidades profesionales, si encajan, refuerza el ambiente de aprendizaje, pero si son discrepantes, pueden empobrecer el clima socioemocional del aula. Así lo expresan maestras y alumnas:

La chica de prácticas era muy rígida, creo que no tenía una personalidad adecuada para desarrollar una metodología de proyectos. (GFM23)

Mira que le he dado oportunidades, que la he dejado hacer, que le he dado confianza... pues no ha logrado meterse a tope en el proyecto. (GFM24)

La maestra no confiaba mucho en los niños... Le proponía algo y me decía: "No, no esto es no lo pueden hacer los niños, es muy difícil para ellos. (MRE11)

Mi maestra estaba muy preocupada por la lectoescritura y por los números. Hacía proyecto poco rato y luego a escribir... Le preocupaba mucho que las familias pensaran que no aprendían los niños a leer y escribir en su clase. (MRE25)

Nuestros hallazgos coinciden con el trabajo de Friend y Cook (2007, p. 29) cuando señalan que "para que funcione la pareja educativa hay que generar la 'cultura del encuentro". Se basa en profesionalizar la práctica y generar debate pedagógico para evitar los problemas personales". 


\section{Conclusiones}

El primer interrogante que focaliza este estudio es: ¿Contribuyen los procesos de investigación-acción colaborativos desarrollados en el Prácticum RIECU a transformar la práctica educativa? Según revelan los datos, los procesos de investigación-acción colaborativos favorecen los procesos de reflexión en y sobre la práctica, disponiendo de un tiempo de sosiego semanal para dialogar profesionalmente y para confrontar la práctica y la teoría. Los datos muestran la potencialidad de los procesos de investigación-acción colaborativos para incorporar miradas externas que contribuyan a objetivar la práctica, a aportar matices que habitualmente son eclipsados por la rutina.

El segundo interrogante incluido en este trabajo hace referencia a: ¿Facilita la documentación pedagógica realizada en el Prácticum RIECU la reflexión en y sobre la práctica? Los datos muestran que la documentación pedagógica es una estrategia de investigación en el aula infantil que ayuda a seleccionar las situaciones relevantes, diferenciándolas de las rutinarias, facilita la captación de detalles del aprendizaje personal de los niños y las niñas, y permite analizar las situaciones de aprendizaje colectivo o la interacción entre iguales.

El tercer interrogante alude a: ¿Propicia la pareja educativa un diálogo profesional para mejorar y transformar la práctica educativa? Los datos identifican como fortalezas de la pareja educativa las alianzas profesionales que propicia. Al establecerse una relación simétrica entre maestra y estudiante, las futuras docentes tienen más espacio para su proyección, para proponer actividades e involucrarse en la vida del aula, que en los formatos tradicionales de Prácticum, en los que prevalece un rol más jerarquizado entre la maestra y la estudiante (Cruger, 2018).

Los resultados revelan que la calidad de la colaboración depende, en gran medida, de la compatibilidad entre la identidad personal y profesional de las profesionales que integran la pareja. De este modo, el buen funcionamiento de cada pareja educativa es proporcional a la armonía, complicidad y afinidad que se cree entre ambas personas. Una limitación de este trabajo es que no se aplica la recomendación sugerida en el trabajo de Cotrina, García y Caparrós (2017, p. 62), donde se indica que "la formación de la pareja no puede ser arbitraria, sino, que ha de ser pensada desde la complicidad entre los miembros, ya que ésta es un ingrediente fundamental”. En el Prácticum RIECU las maestras y las estudiantes no se conocen con anterioridad, por lo que resulta difícil valorar el grado de compatibilidad que poseen previamente. Como prospectiva de esta investigación se plantea la necesidad de establecer criterios para mejorar la compatibilidad pedagógica y personal de las parejas educativas, con el fin de facilitar la 'cultura del encuentro'.

Para concluir, podemos afirmar que el Prácticum RIECU permite a los futuros y futuras docentes repensar los procesos y los contextos educativos en los que participan, desarrollar procesos de reflexión compartida y ofrecer espacios de diálogo que actúan como palancas de transformación y mejora de la práctica.

\section{Referencias}

Adorno, T. (1991). La actualidad de la filosofía. Barcelona: Paidós.

Altimir, D. (2010). Cómo escuchar a la infancia. Madrid: Octaedro. 
Álvarez, C. (2015). Teoría frente a práctica educativa: Algunos problemas y propuestas de solución. Perfiles Educativos, 37(148), 172-190.

https://doi.org/10.22201/iisue.24486167e.2015.148.49320

Arnaus, R. y Piussi, A. M. (2010). La universidad fértil. Mujeres y hombres. Una apuesta política. Barcelona: Octaedro.

Barnett, R. (2002). Claves para entender la universidad en una era de supercomplejidad. Barcelona: Pomares.

Barnett, R. (Ed.) (2008). Para una transformación de la universidad. Nuevas relaciones entre investigación, saber y docencia. Barcelona: Octaedro.

Boggino, N., y Rosekrans, K. (2007). Investigación-acción: Reflexión crítica sobre la práctica educativa. Orientaciones prácticas y experiencias. Rosario: Homo Sapiens.

Brydon-Miller, M., Greenwood, D. y Maguire, P. (2003). Why action research? Action Research, 1(1), 9-28. https://doi.org/10.1177/14767503030011002

Calvo, A. y Susinos, T. (2010). Prácticas de investigación que escuchan la voz del alumnado: Mejorar la universidad indagando la experiencia. Profesorado, Revista de Currículum y Formación del Profesorado, 14(3), 75-88.

Carr, W. (2002). Una teoría para la educación. Hacia una investigación educativa crítica. Madrid: Morata.

Chanmugam, A. y Gerlach, B. (2013). A co-teaching model for developing future educators' teaching effectiveness. International Journal of Teaching and Learning in Higher Education, 25(1), 10-117.

Colmenares, A. M. (2012). Investigación-acción participativa: Una metodología integradora del conocimiento y la acción. Voces y Silencios: Revista Latinoamericana de Educación, 3(1), 102115. https://doi.org/10.18175/vys3.1.2012.07

Colmenares, A. y Piñero. M. L. (2008). La investigación-acción. Una herramienta metodológica heurística para la comprensión y transformación de realidades y prácticas socio-educativas. Laurus. Revista de Educación, 14(27), 96-114. https://doi.org/10.18356/1458bfa5-es

Cotrina, M., García, M. C. y Caparrós, E. (2017). Ser dos en el aula. Las parejas pedagógicas como estrategia de co-enseñanza inclusiva en una experiencia de formación inicial del profesorado de secundaria. Aula Abierta, 46(0), 57-64. https://doi.org/10.17811/rifie.46.2017.57-64

Cruger, K. M. (2018). Applying challenge-based learning in the (feminist) communication classroom: Positioning students as knowledgeable change agents. Communication Teacher, 32(2), 87-101. https://doi.org/10.1080/17404622.2017.1372602

Denzin, N. K. (2017). The research act. A theoretical introduction to sociological methods. Nueva York, NY: Routledge. https://doi.org/10.4324/9781315134543

Equipo Directivo y Educativo de la EEI La Alegría de la Huerta. (2018). La documentación como herramienta pedagógica en una escuela infantil. Recuperado de http://comunidad.educa.madrid.org/redformacion/tag/documentacion/

Espín, J. V. (2002). El Análisis de contenido una técnica para explorar y sistematizar información. XXI. Revista de Educación, 4, 95-106.

Farrell, T.S.C. (2018). Research on reflective practice in TESOL. Nueva York, NY: Routledge. https://doi.org/10.4324/9781315206332

Friend, M., y Cook, L. (2007). Interactions: Collaboration skills for school professionals. Boston, MA: Pearson Education Inc. 
Friend, M., Cook, L., Hurley-Chamberlain, D. y Shamberger, C. (2010). Co-teaching: An illustration of the complexity of collaboration in special education. Journal of Educational and Psychological Consultation, 20(2), 9-27. https://doi.org/10.1080/10474410903535380

Habermas, J. (1999). Teoría de la acción comunicativa I. Madrid: Taurus.

Horkheimer, M. (2000). Teoría tradicional y teoría crítica. Barcelona: Paidós.

Hoyuelos, A. (2004). La pareja educativa: Un reto cultural. Infancia, 86, 4-10.

Hoyuelos, A. (2006). La estética en el pensamiento y obra pedagógica de Loris Malaguzzi. Barcelona: Octaedro.

Hoyuelos, A. (2007). Documentación como narración y argumentación. Aula de Infantil, 39, art 3.

Inayatullah, S. y Gidley, J. (2003). La universidad en transformación. Perspectivas globales sobre los futuros de la universidad. Barcelona: Pomares.

Kemmis, S. (2002). La teoría de la práctica educativa. En W. Carr (Ed.), Una teoría para la educación. Hacia una investigación educativa crítica, (pp. 17-32). Madrid: Morata.

Korthagen, F. (2010). Situated learning theory and the pedagogy of teacher education: Towards an integrative view of teacher behavior and teacher learning. Teaching and Teacher Education, 26(1), 98-106. https://doi.org/10.1016/j.tate.2009.05.001

Krichesky, G. y Murillo, F. J. (2011). Las comunidades profesionales de aprendizaje. Una estrategia de mejora para una nueva concepción de escuela. REICE. Revista Iberoamericana sobre Calidad, Eficacia y Cambio en Educación, 9(1), 65-83.

Krueger, R. A. (1994). Focus groups: a practical guide for aplied research. Thousand Oaks, CA: SAGE.

Mérida, R., González, E. y Olivares, M. A. (2012). RIECU: Una experiencia de innovación en el prácticum I del grado de infantil de la Universidad de Córdoba. Profesorado. Revista de Currículum y Formación del Profesorado, 16(3), 447-465.

Miño Puigcercós, R., Domingo-Coscollola, M. y Sancho-Gil, J. (2019). Transforming the teaching and learning culture in higher education from a DIY perspective. Educación XX1, 22(1), 139-160. https://doi.org/10.5944/educxx 1.20057

Oliveros, E. (2018). Centros educativos transformadores. Rasgos y propuestas para avanzar. Madrid: Ministerio de Asuntos Exteriores y AECID.

Pérez, A. I. (2012). Educarse en la era digital. Madrid: Narcea.

Perrenoud, P. (2004). Desarrollar la práctica reflexiva en el oficio de enseñar. Racionalizar la profesionalización y razón pedagógica. Barcelona: Graó.

Rinaldi, C. (2011). En diálogo con Reggio Emilia. Lima: Grupo Editorial Norma.

Rivas, J. I., Hernández, F., Sancho, J. M. y Núñez, C. (2012). Historias de vida en educación: Sujeto, diálogo, experiencia. Barcelona: Dipòsit Digital UB.

Rivera-Vargas, P., Sánchez-Valero, J. A., y Sancho-Gil, J. M. (2019). La cultura DIY en la universidad. De la propuesta del profesorado a la experiencia del alumnado. REIRE Revista d'Innovació $i$ Recerca en Educació, 12(1), 1-13.

Rodríguez, E., y Grilli, J. (2013). La pareja pedagógica: Una estrategia para transitar y aprender el oficio de ser profesor. Páginas de Educación, 6(1), 61-81.

Ruiz, P., Sánchez-Tarazaga, V. y Mateu-Pérez, R. (2018). La innovación pedagógica de la mano de la investigación-acción para mejorar la calidad del prácticum de magisterio. Revista Electrónica Interuniversitaria de Formación del Profesorado, 21(1), 33-49. https://doi.org/10.6018/reifop.21.1.277681 
Salamanca, C. (2011). Dos docentes, dos miradas: la pareja educativa. Tarbiya, Revista de Investigación e Innovación Educativa, 42, 95-102.

Sancho-Gil, J. M. (2017). Discursos y prácticas en torno a las competencias en educación. Fonseca, Journal of Communication, 15, 127-144. https://doi.org/10.14201/fjc201715127144

Schön. D. (1992). La formación de profesionales reflexivos. Hacia un nuevo diseño de la enseñanza y el aprendizaje en las profesiones. Barcelona: Paidós.

Suárez, M. (2002). Algunas reflexiones sobre la investigación-acción colaboradora en la educación. Revista Electrónica de Enseñanza de las Ciencias, 1(1), 40-56.

Urdaneta, M. M. (2014). Diálogo para la reflexión: Compartiendo la experiencia de aula desde el proyecto pedagógico. Innovaciones Educativas, 16(21), 43-49. https://doi.org/ 10.22458/ie.v16i2 1.902

Whitehead, J. (2008). Using a living theory methodology in improving practice and generating educational knowledge in living theories. Educational Journal of Living Theories, 1(1), 103126.

Wodak, R. (2000). ¿La sociolingüística necesita una teoría social? Nuevas perspectivas en el análisis crítico del discurso. Revista Iberoamericana de Discurso y Sociedad, 2(3), 123-147.

Woolf, S. B. y Wamba, N. G. (2019). Embracing intersectionality to create a collective living theory of practice. Action Research, $17(2)$ 208-219. https://doi.org/10.1177/1476750318818879

Zamboni, C. (2009). Sobre la universidad y sus necesidades. DUODA. Estudis de la Diferència Sexual, $36,125-129$.

Zeichner, K. (2010). New epistemologies in teacher education. Rethinking the connections between campus courses and field experiences in college and university-based teacher education. Revista Interuniversitaria de Formación del Profesorado, 68, 123-149.

Zeichner, K. M. y Liston, D. P. (2013). Reflective teaching. An introduction. Nueva York, NY: Routledge. https://doi.org/10.4324/9780203771136

\section{Breve CV de los autores}

\section{Rosario Mérida Serrano}

Doctora en Psicopedagogía y catedrática de Didáctica y Organización Escolar del Departamento de Educación de la Universidad de Córdoba. Sus líneas de investigación más relevantes son la formación docente inicial y continua, la educación infantil, la convivencia y la investigación cualitativa. Ha participado y coordinado proyectos de ámbito nacional y local. Posee publicaciones en revistas de impacto nacionales e internacionales. Es docente del Máster de Educación del Profesorado de Secundaria y del Máster de Educación Inclusiva, participando en el itinerario de género. Es coordinadora del grupo de investigación INCIDE, SEJ-614. ORCID ID: https://orcid.org/0000-00019389-3263. Email: ed1meser@uco.es

\section{Ma Elena González Alfaya}

Licenciada en Pedagogía y doctora europea por la Universidad de Santiago de Compostela (España). Actualmente es profesora del departamento de Educación de la Universidad de Córdoba. Imparte clase en el Grado de Educación Infantil y en el itinerario de género del Máster de Educación Inclusiva. Sus líneas de investigación más relevantes son la didáctica 
de contenidos transversales (en especial la educación para la salud), la educación infantil, la convivencia e interculturalidad y la innovación educativa. Ha participado en proyectos de ámbito internacional, nacional y local. Participa en el grupo de investigación INCIDE, SEJ-614. Posee publicaciones en revistas nacionales e internacionales relacionadas con las líneas de investigación descritas. ORCID ID: https://orcid.org/0000-0001-8264-3680. Email: ed2goalm@uco.es

\section{Ma Ángeles Olivares García}

Maestra de Educación Infantil, licenciada en Psicopedagogía y doctora. Actualmente es profesora del departamento de Educación de la Universidad de Córdoba, en la que imparte docencia en el Máster de Educación Inclusiva, en el itinerario de Género y en el Máster Universitario en Participación y Desarrollo desde una Perspectiva de Género. Ha coordinado diversos proyectos de transferencia y ha participado en proyectos de investigación de ámbito nacional y autonómico. Sus líneas de investigación más relevantes son la formación docente inicial y continua, la educación infantil, la formación y orientación para el empleo y la educación de personas adultas. Participa en el grupo de investigación INCIDE, SEJ-614. ORCID ID: https://orcid.org/0000-0002-9440-5628. Email: ed1olgam@uco.es

\section{Julia Rodríguez Carrillo}

Graduada en Educación Infantil por la Universidad de Córdoba, ha cursado el máster en Educación Inclusiva, ofertado por la misma Universidad, siguiendo la línea de Educación Intercultural. Actualmente, es becaria de Formación del Profesorado Universitario (FPU) en el Departamento de Educación. Realiza su tesis sobre la percepción de la comunidad educativa sobre la excelencia docente en Educación Infantil. Ha disfrutado de una estancia en EEUU, desarrollando su trabajo de campo en dos colegios de Washington DC y ha participado en el Project Zero de la Universidad de Harvard. Ha realizado múltiples cursos de formación en metodología de investigación cualitativa y cuantitativa y ha participado en congresos científicos de carácter nacional e internacional. Participa en el grupo de investigación INCIDE, SEJ-614. ORCID ID: https://orcid.org/0000-0003-0725-3054. Email: juroca93@gmail.com

\section{Miguel Muñoz Moya}

Diplomado por la Universidad de Córdoba en Magisterio de Educación Primaria (2012) y Máster en Educación Inclusiva (2013) en el itinerario de Educación Intercultural. Desde 2013 soy colaborador honorario del departamento de Educación en la Universidad de Córdoba. Ha realizado complementos formativos en los ámbitos de Educación Intercultural, Convivencia y Cultura de Paz, y Estudios de Género e Igualdad. Ha formado parte del proyecto financiado por la FECYT "Infaciencia, de las niñas de hoy a las científicas de mañana”. Actualmente está contratado con cargo al grupo de investigación INCIDE (Infancia, Ciudadanía y Democracia) SEJ-614. Actualmente realiza su tesis doctoral en la formación inicial docente. Ha participado en múltiples cursos de formación en metodología de investigación cualitativa y cuantitativa y ha participado en congresos científicos de carácter nacional e internacional. ORCID ID: https://orcid.org/0000-00016250-2995. Email: miguelmunoz88@gmail.com 